\title{
Lumen
}

Selected Proceedings from the Canadian Society for Eighteenth-Century Studies

\section{Aller-retour : fonction épistémologique et reflexive de l'altérité chez le Diderot de l'Encyclopédie}

\section{Mitia Rioux-Beaulne}

Volume 24, 2005

Indigenes and Exoticism

Indigènes et exotisme

URI : https://id.erudit.org/iderudit/1012176ar

DOI : https://doi.org/10.7202/1012176ar

Aller au sommaire du numéro

Éditeur(s)

Canadian Society for Eighteenth-Century Studies / Société canadienne d'étude du dix-huitième siècle

ISSN

1209-3696 (imprimé)

1927-8284 (numérique)

Découvrir la revue

Citer cet article

Rioux-Beaulne, M. (2005). Aller-retour : fonction épistémologique et reflexive de l'altérité chez le Diderot de l’Encyclopédie. Lumen, 24, 79-91.

https://doi.org/10.7202/1012176ar

Copyright (C Canadian Society for Eighteenth-Century Studies / Sociéte canadienne d'étude du dix-huitième siècle, 2005
Ce document est protégé par la loi sur le droit d'auteur. L'utilisation des services d'Érudit (y compris la reproduction) est assujettie à sa politique d'utilisation que vous pouvez consulter en ligne.

https://apropos.erudit.org/fr/usagers/politique-dutilisation/ 


\section{Aller-retour : fonction épistémologique et réflexive de l'altérité chez le Diderot de l'Encyclopédie}

\section{Préambule}

Ce texte est le fruit d'une analyse d'un certain groupe d'articles écrits par Diderot pour l'Encyclopédie. Le groupe d'articles en question était formé de tous ceux dont la rubrique contenait le terme "géographie», de tous ceux appartenant à $l^{\prime}$ «histoire de la philosophie», ainsi qu'un certain nombre d'autres $d^{\prime}$ '«histoire moderne» et d' $\mathrm{d}^{\prime}$ histoire ancienne» (un total de plus de 1500 articles). Une attention particulière a été portée sur ceux dont l'entrée était formée du nom d'un peuple. Il va sans dire que nombre de ces articles offraient peu d'intérêt : tous ceux ne donnant que la latitude et la longitude d'un lieu, par exemple, et qui constituent, au fond, la majorité de ceux appartenant au groupe «Géographie moderne». Les articles cités ici le sont à partir du fac-similé de l'édition originale (Paris, Briasson, 1751-1765), publié à Stuttgart, F. Frommann Verlag, 1966, mais le texte a été confronté, quand son équivalent s'y trouvait, avec l'édition des CEuvres complètes de Diderot qui est en cours chez Hermann (sous la direction de H. Dieckmann, J. Proust et J. Varloot). L'attribution des textes à Diderot à été vérifiée à partir de l'Annexe II du livre essentiel de J. Proust, Diderot et l'Encyclopédie, Paris, Armand Colin, 1967, et de l'Inventory of Diderot's Encyclopédie, établi par R. N. Schwab, W. E. Rex et J. Lough, Studies on Voltaire and the Eighteenth Century, vol. 80, 83, 85, 91, 92, 93, 1971-1972.

\section{Introduction}

«On ne cite des faits, écrit Diderot dans le Prospectus de l'Encyclopédie, on ne compare des expériences, on n'imagine des méthodes, que pour exciter le génie à s'ouvrir des routes ignorées, et à s'avancer à des découvertes nouvelles, en regardant comme le premier pas celui où les 
grands hommes ont terminé leur course ${ }^{1} . » C^{\prime}$ est là ce qui aura formé le fondement de cet «esprit» des Lumières tel que Diderot le comprenait, à savoir le fait d'être un pivot ou un tremplin pour le "génie», d'être un moment clé où la pensée, contre la stagnation que sont pour elle la superstition et le dogmatisme, se met en activité. Pour Diderot, la vérité de la pensée est son mouvement même, comme ce mouvement est, en dernière analyse, la vérité de toute la nature. Dès lors, «exciter le génie» aura toujours été, en un certain sens, une manière de restituer la pensée à sa nature même. Et le travail accompli pour l'Encyclopédie par Diderot, à n'en pas douter, a été pour lui, ou aurait dû être, une réalisation pratique de ce programme. Citer des faits, donc, comparer des expériences ou imaginer des méthodes pour ouvrir des routes ignorées ne correspondait pas pour Diderot simplement à élargir le champ d'une connaissance s'accumulant et se reposant sur ses propres acquis, mais aussi à s'assurer que cette connaissance agisse, pour ainsi dire, rétroactivement sur ces acquis d'une manière destructive, l'empêchant d'être une force d'inertie pour elle-même. Ce que confirme, entre autres, la théorie des "renvois» de l'article Encyclopédie : «Ils opposeront les notions ; ils feront contraster les principes ; ils attaqueront, ébranleront, renverseront secrètement quelques opinions ridicules qu'on n'oserait insulter ouvertement. Si l'auteur est impartial, ils auront toujours la double fonction de confirmer et de réfuter ; de troubler et de concilier», et ce, jusqu'à "changer la façon commune de penser ${ }^{2}$.»

$C^{\prime}$ est là une articulation particulière du discours encyclopédique que nous présente Diderot où, au premier emploi que l'Encyclopédie semble s'être assignée - celui d'être "un sanctuaire où les connaissances des hommes soient à l'abri des temps et des révolutions ${ }^{3}$ " - $\mathrm{s}^{\prime}$ oppose une utilisation beaucoup plus réflexive de la connaissance où, cette fois, l'objectif semble être de propager dans le lectorat l'attitude même qu'a supposé l'établissement de cette connaissance, c'est-à-dire une capacité de neutraliser la connaissance faisant autorité. Diderot constate que, dans le développement du savoir, le moment le plus dangereux est celui où une connaissance est tenue pour vraie et propagée comme telle par

1 Versini, I, p. 218. (Outre les références aux articles de l'Encyclopédie, les renvois à l'œuvre de Diderot suivront l'édition établie par L. Versini, CEuvres (5 volumes), Paris, Robert Laffont, 1994-1997, notés de la manière suivante : Versini, [No. de volume], [No. de page].)

2 Versini, I, p. 402-403.

3 Prospectus, Versini, I, 220. 
ceux qui se confèrent une autorité pour l'avoir découverte ou pour en maîtriser l'outil privilégié de propagation, à savoir le discours, puisque ce moment est le point précis où le savoir tourne en superstition ou en dogmatisme.

Le champ «ethnographique», comme beaucoup d'autres des recherches de Diderot pour l'Encyclopédie, permet de voir comment cette manière de mobiliser et de neutraliser dans un même geste le savoir de son temps ouvre un espace réflexif faisant barrage à la tendance de la connaissance à s'instituer en superstition. "Champ ethnographique» doit s'entendre en un sens large. Cela recoupe les articles écrits par Diderot que l'on retrouve tout particulièrement sous les classifications géographie, histoire ancienne, histoire moderne, et histoire de la philosophie. Toutes ces classifications donnent lieu à une interrogation que l'on qualifierait aujourd'hui d'ethnographique, dans la mesure où Diderot $y$ décrit des éléments culturels, le plus souvent sur la base de divisions ou de comparaisons nationales ou ethniques. C'est dans le cadre de cette interrogation que Diderot met en œuvre un certain nombre de pratiques scripturales faisant intervenir la problématique de l'altérité ethnologique. Ou plutôt, Diderot y problématise cette altérité en tant que telle, et ce, en travaillant selon ces deux axes qui sont ceux de la science de son temps : un constat empirique de la diversité des phénomènes - ici les mœurs - motivant une approche de type ethnologique, et l'assurance profonde que des principes généraux sont au fond de toute cette diversité, donnant alors lieu à une sorte d'anthropologie philosophique universalisante.

Il y a, essentiellement, trois modes discursifs mis en œuvre par Diderot dans ses articles ethnographiques. Ces trois modes discursifs ouvrent sur trois types de problèmes relatifs à l'altérité. Le premier d'entre ces modes fait jouer une critique épistémologique des récits sur les mœurs des autres nations. Le deuxième prend acte de la diversité des mœurs et tente d'en dégager une explication par le recours à une historicisation. Le troisième, enfin, met en rapport les histoires particulières comme autant de parallèles, ce qui permet la formulation d'une loi historico-anthropologique expliquant cette diversification. Mon objectif, ici, est de montrer comment l'élaboration de ces trois modes discursifs permet à Diderot d'ouvrir ce champ réflexif sur le dynamisme duquel il compte pour maintenir la pensée en mouvement.

\section{Localisation de l'altérité}

Les relations de voyage et les descriptions ethnographiques forment l'une des catégories sur lesquelles Diderot, comme la plupart des phi- 
losophes de son temps ${ }^{4}$, laisse planer les doutes les plus sévères, en ce qui concerne la fiabilité. L'article "Ansico» est à cet égard des plus éloquents. Après avoir rappelé que le Dictionnaire géographique de Vosgien accuse les habitants de ce royaume d'Afrique de prendre pour un honneur que de servir de repas pour le roi, Diderot reste dubitatif. «Plus [les] circonstances sont extraordinaires, écrit-il, plus il faudra de témoins pour les faire croire», ajoutant qu'«il faut soupçonner en général tout voyageur et tout historien d'enfler un peu les choses... on ne veut pas avoir pris la plume pour raconter des aventures communes, ni fait des milliers de lieues pour n'avoir vu que ce qu'on voit sans aller si loin ${ }^{5} . »$ Sur ce soupçon, Diderot dit qu'il pourrait presque assurer que ce barbarisme est de pure invention.

Ce qui est notoire, dans cette manière de présenter les habitants d'Ansico, c'est qu'à la fin de l'article, la seule chose que l'on puisse dire de ce peuple est qu'il existe quelque part en Afrique et qu'il est peut-être anthropophage. Autrement dit, Diderot, par le détour d'une critique épistémologique du témoignage en général, empêche l'institution de ce témoignage en fait, pour ensuite lui redonner droit de cité dans la dimension du possible. Cette manière de traiter les récits sur les mœurs des autres nations est typique dans l'écriture diderotienne : une forte concentration d'articles relatant les mœurs des diverses nations non européennes utilise pareille stratégie, tantôt discrètement, faisant simplement précéder la description d'un "on dit que...", tantôt directement en mettant en question la validité du témoignage ${ }^{6}$.

4 Voir Michèle Duchet, Anthropologie et histoire au siècle des Lumières, Paris, Albin Michel, 1995, «La critique des relations de voyages et l'évolution du genre», 95-113, où l'auteure écrit, notamment, que les philosophes font preuve de scepticisme quant à ce genre d'écrits «moins par un mépris systématique que par un doute méthodique : il n'y a pas pour eux de différence entre la littérature des voyages et les textes sacrés, les récits des anciens historiens, ou les compilations des érudits qui servent de matériaux à la connaissance historique.» (p. 99)

5 On trouvera presque la même phrase dans le Supplément au Voyage de Bougainville. Voir Versini, II, p. 544-545.

6 Voir, par exemple, les articles «Ardée», «Batuecas», «Babylone», «Brésil», «Castalie», «Dydime», pour ne nommer qu'eux. On ne peut omettre de mentionner l'article «Besançon» qui fait intervenir le même genre de développement en ironisant sur le fait qu'une grotte près de cette ville a fait l'objet du même genre de témoignages absurdes que si elle s'était trouvée en Cochinchine. Évidemment, Diderot n'utilise pas cette stratégie dans les seuls articles à caractère ethnographique : le cas de l'article «Agnus Scythicus» est bien connu, où il prend prétexte de la description d'une plante pour élaborer une théorie sur la mesure de la validité des témoignages. 
L'intérêt du procédé est multiple. Certes, il permet-ce qui correspond au programme encyclopédique - de rendre compte de l'état actuel de la connaissance sur les mœurs, et le nombre d'entrées ethnographiques à caractère purement anecdotique montre qu'il y va d'une sorte d'inventaire de la diversité du genre humain. Mais il a aussi la vertu de rappeler le caractère provisoire et partiel de cette connaissance, par une mise en lumière du réseau complexe de contingences auquel elle est soumise. Et à ce réseau n'appartient pas que la vanité des voyageurs et des historiens, mais aussi une multitude d'autres facteurs comme le patriotisme de ceux qui témoignent, qui les pousse à glorifier leur nation et à dévaloriser les autres, leur crédulité, leur soumission à l'autorité plutôt qu'à l'expérience, le manque de vestiges ou de documents, l'authenticité douteuse de ceux que l'on possède encore et l'interprétation que l'on en fait, qui relève plus souvent qu'autrement de la «rêverie» ${ }^{7}$.

On peut ici rappeler que ces remarques épistémologiques sont exactement du même ordre que celles qu'il adresse aux philosophes de la nature dans les Pensées sur l'interprétation de la nature, à savoir que la nature ne se donne jamais que par fragments et que la formation d'une vue unitaire sur celle-ci n'est jamais qu'un processus conjectural de reconstruction. On doit de même rappeler que ce scepticisme à l'égard des récits fait partie du réseau complexe de critique scripturaire de la Bible entamé à la fin du XVII ${ }^{\mathrm{e}}$ siècle et au début du XVIII ${ }^{\mathrm{e}}$ siècle par les Spinoza, Bayle et autres, critique qui s'est par la suite étendue à tous les témoignages historiques tant en France, chez des gens comme Nicolas Fréret, qu'en Angleterre, chez Hume, par exemple. ${ }^{8}$ Il s'agit toujours, pour ces critiques, de montrer que les discours sur l'histoire ne sont pas l'histoire même, que l'histoire, donc, n'est jamais épuisée par sa description, et qu'elle peut faire l'objet d'une reconstruction pleinement valable seulement a posteriori.

En ce qui a trait à l'altérité ethnologique, Diderot fait fonctionner le même système de critique. Cette altérité n'est jamais épuisée dans sa description dans la stricte mesure où celle-ci s'aperçoit à partir de signes visibles qu'elle donne d'elle-même, signes qui sont toujours fragmen-

7 Voir notamment les articles «Baaras», «Chaldéens», ainsi que tous ceux compris sous la rubrique «Histoire de la philosophie».

8 Pour Hume, voir le Treatise of Human Nature, Livre I, $3^{\mathrm{e}}$ partie, section 13 (Of unphilosophical probability); pour Fréret, les Réflexions sur l'étude des anciennes histoires et sur le degré de certitude de leurs preuves du 17 mars 1724 (dans Mémoires académiques, Paris, Fayard, 1996). 
taires ${ }^{9}$. Pousser cette idée un pas plus loin implique en définitive que l'altérité n'est jamais l'objet du savoir en tant que tel : ce que l'on vise alors, c'est bien plutôt le décalage historique sauvagerie/civilisation, en tant qu'il permet de caractériser l'identité humaine. A l'inverse, cela implique également que toute prétention à détenir un savoir sur l'autre opère une réduction de cette altérité, ce qui est aussi une négation de sa singularité. La manière typiquement diderotienne de sortir de ce paradoxe consiste à jouer sur les deux registres de l'effectif et du possible. L'effectif correspond alors à la diversité observable des phénomènes, ce qui signifie, sur le plan ethnologique, la diversité des mœurs. Faire un inventaire des mœurs observées dans les différentes nations permet à Diderot de mobiliser la connaissance pour affirmer le caractère multiple de la réalité effective. De ce point de vue, Diderot joue sur l'éloignement qu'il y a entre les formes culturelles, s'intéressant, comme il le fait pour la biologie, aux cas-limites qui manifestent la plus grande étrangeté. Le registre du possible, quant à lui, correspond à la formation de l'unité non observable qui se cache au fond de toute cette diversité, l'espèce. En ce qui a trait aux phénomènes ethnologiques, Diderot tente de rejoindre les conditions de possibilité d'un développement aussi diversifié de l'humanité. Seulement, il prend au pied de la lettre le fait que ce registre est celui du possible.

\section{Altérité de l'autre}

Ces dernières remarques demandent des éclaircissements, et une analyse de ce quej'ai annoncé comme un deuxième mode discursif employé par Diderot dans les articles à caractère ethnographique devrait pouvoir les fournir. L'idée, ici, est que, si l'on applique rigoureusement l'épistémologie diderotienne, on doit assumer que l'altérité ne s'entend que comme une intériorité, intériorité qui, explique la Lettre sur les aveugles, ne peut faire l'objet d'une induction. ${ }^{10}$ Autrement dit, on doit

9 Michèle Duchet (op. cit., «Le rôle des naturalistes», 114-125) a bien montré que l'image que l'on avait au XVIII ${ }^{\mathrm{e}}$ siècle du Nouveau monde était partielle. Or les naturalistes et les encyclopédistes manifestent justement ce changement d'attitude qui a permis la formation de l'anthropologie en s'intéressant à reconstruire une image la plus fidèle possible du «sauvage» à partir des signes disponibles de son mode de vie — intérêt marqué pour les instruments et outils, les descriptions physiques, les dessins, les alphabets, etc.

10 Versini, I, p. 141. Diderot écrit que le commerce avec un aveugle «ôterait beaucoup 
assumer que le travail d'interprète de la nature qui est déposé entre les mains du philosophe est un travail qui consiste à produire une vue unifiée des conditions de possibilité de surgissement de cette altérité. Ce qui signifie, pour Diderot, une remontée aux causes possibles de ce qui est ${ }^{11}$.

S'il est vrai que, en ce qui concerne la nature, cette remontée implique une enquête comme celle entreprise par Buffon et Daubenton, c'est-àdire une reconstruction de l'histoire de la nature, les articles ethnographiques de Diderot montrent que sur le plan anthropologique, c'est ce même modèle qu'il faudra employer ${ }^{12}$. Les articles dits d'histoire ancienne, d'histoire moderne et d'histoire de la philosophie illustrent qu'un procédé semblable à celui employé dans la Lettre sur les aveugles, où Diderot tente de reconstruire la philosophie d'un aveugle à partir d'une genèse sensualiste, peut permettre une approche de la «philosophie» des autres nations. Et cette genèse sera historique. L'histoire des nations devient ainsi un horizon de compréhension de leur singularité. L'altérité ethnologique doit être vue non pas comme une détermination d'essence, mais comme le produit d'un développement. Et ce qui intéresse Diderot au plus haut point, c'est d'expliquer comment cet autre est devenu ce qu'il est $^{13}$.

À ce titre, donc, la séparation identifiée plus tôt des registres de l'effectif et du possible ressurgit. C'est que devant le constat d'une

de force à cette induction que nous faisons tous, sans savoir pourquoi, de ce qui se passe en nous à ce qui se passe au-dedans des autres.»

11 Ce schéma correspond globalement à l'épistémologie que développe Diderot dans les Pensées sur l'interprétation de la nature, contemporaines de son travail pour l'Encyclopédie - voir notamment les pensées 1, 6, 7, 9, 14, 15, 20, 23 et, surtout, 56 : l'interprète de la nature s'élève, par des conjectures, «à l'essence même de l'ordre», mais ne porte pas de «jugement absolu».

12 Michèle Duchet (op. cit., p. 479) écrit : «Chez tous les philosophes, le point de vue historique l'emporte sur le point de vue anthropologique ; l'objet principal de leur science de l'homme est de combler les lacunes et les silences de l'histoire, pour les siècles qui séparent l'homme des origines de l'invention de l'écriture.»

13 Évidemment, le système de catégories historiques suppose aussi que cet autre est placé dans un état antérieur à celui de la civilisation européenne. Mais on sait que Diderot a pour idéal une société qui serait à mi-chemin entre sauvagerie et civilisation, ce qui signifie que l'antériorité n'a pas exactement un sens péjoratif ni, en contrepartie, un sens unilatéralement positif. Diderot semble plutôt s'intéresser aux diverses conditions qui ont produit tel ou tel aspect singulier d'une nation afin de cerner les différents facteurs historiques qui sont susceptibles d'affecter le développement des sociétés. 
diversité effective, Diderot tente de faire jouer une histoire possible qui rendrait compte de cet état de fait. Cette histoire possible ou conjecturale, lorsque l'on compare les articles où elle intervient - c'est tout particulièrement le cas de ceux classés sous la rubrique «Histoire de la philosophie» - fait osciller la pensée de Diderot entre deux lignes argumentatives : l'une est de type historique proprement dit et permet de saisir les rudiments de ce que l'on pourrait appeler une philosophie diderotienne de l'histoire; l'autre, qui sert aussi de soubassement à la première, est de type anthropologique et suggère que Diderot cherche à formuler un certain nombre de lois générales définissant les limites de ce que l'on peut bien appeler une nature humaine, pour autant que cela ne soit pas pris en un sens essentialiste. La nature humaine ne circonscrit pas les possibles donnés à l'être humain ni ses fins, mais les conditions de possibilité de sa diversification à l'infini - ce qui est aussi le concept de la nature entière pour Diderot : «Il semble, écrit-il, que la nature se soit plu à varier le même mécanisme d'une infinité de manières différentes ${ }^{14}$.»

La première ligne argumentative, celle dite historique, est une interprétation qui vise à expliquer comment un peuple en est venu à avoir telle ou telle "philosophie», à développer ses mœurs propres. Il s'agit, dans ce contexte, de raconter l'histoire particulière de chaque peuple et d'essayer de comprendre la formation de ce qu'aujourd'hui nous appellerions peut-être sa «culture». Pour le contenu de ces articles, Diderot emprunte beaucoup à Pierre Bayle et à Jackob Brucker ${ }^{15}$. Mais ce qui $\mathrm{m}^{\prime}$ intéresse plus particulièrement ici, $c^{\prime}$ est la manière dont Diderot traite ce contenu, la forme, pour ainsi dire, des articles, forme qui manifeste l'enjeu épistémologique dont il est question, à savoir, comme cela a été dit plus tôt, la manière dont Diderot mobilise et neutralise la connaissance qui lui est accessible. Or, ce qui est prégnant dans sa manière de raconter l'histoire des nations, $c^{\prime}$ est le recours constant qu'il fait au mode conjectural. Qu'il s'agisse de l'origine d'un mythe particulier, de l'émergence d'une science ou d'un système philosophique, Diderot nie toujours qu'on puisse parler de savoir quant à cette origine et propose, en

14 Pensées sur l'interprétation de la nature, pensée 12, Versini, I, p. 564-565.

15 On trouvera en annexe du livre de Jacques Proust Diderot et l'Encyclopédie (Paris, Armand Colin, 1967) une table des correspondances avec les auteurs-sources de Diderot pour ces articles. Dans le chapitre VIII du même livre, l'auteur indique que la manière particulière à Diderot de «fabriquer» ses articles pour l'Encyclopédie (remaniement de l'ordre des paragraphes, ajouts, retranchements, etc.) autorise à les lire comme une expression de sa pensée, pour autant que l'on use de prudence. 
contrepartie, une ou plusieurs conjectures dont il assume la valeur purement conjecturale.

Une remarque s'impose ici. Elle concerne justement le fait, dûment noté par Diderot, que tous les peuples ont la prétention de connaître leur propre origine et cherchent à la faire remonter le plus loin possible, ce qui leur conférerait une certaine autorité ${ }^{16}$. Dans l'article «Chaldéens» il écrit : «L'envie de passer pour les plus anciens peuples du monde, est une manie qui a été commune à toutes les nations. On dirait qu'elles s'imaginent valoir d'autant mieux, qu'elles peuvent remonter plus haut dans l'antiquité. On ne saurait croire combien de rêveries et d'absurdités ont été débitées à ce sujet.» Dès lors, une critique épistémologique s'impose pour montrer le caractère non assuré, voire faux de cette prétendue connaissance de l'origine. Cette critique de l'originarité a un double effet : elle permet de désancrer toute "philosophie nationale» de son foyer originaire et de lui enlever ainsi l'autorité que son appartenance à l'origine pourrait lui conférer ; en même temps, elle permet de «désessentialiser» toute singularité nationale par une dénégation des revendications d'appartenance à une chaîne causale indépendante ${ }^{17}$. La diversité ethnologique tient à une certaine historicité de l'existence humaine, elle-même comprise dans une histoire encore plus générale, une histoire de la nature au sens large. Diderot semble donc envisager l'idée d'une histoire universelle des nations correspondant à sa philosophie matérialiste qui envisage tout autant la formation des espèces comme le produit d'une vicissitude perpétuelle des êtres ${ }^{18}$.

Sur ce plan, on notera la solidarité qu'il y a entre les articles sur les philosophies des Égyptiens, des Chaldéens, des Éthiopiens et des Grecs,

16 Voir les articles «Bédouins», «Arabes», «Chaldéens», «Chinois», «Égyptiens», "Éthiopiens», etc.

17 On se souviendra que les Pensées sur l'interprétation de la nature affirmaient : «L'indépendance absolue d'un seul fait est incompatible avec l'idée de tout ; et sans l'idée de tout, plus de philosophie.» (voir la pensée 11)

18 On notera que cette inscription de l'histoire humaine dans l'horizon plus vaste d'une histoire naturelle favorise une approche plus scientifique que celle, plus sentimentale, que l'on retrouve chez un Rousseau, par exemple. (Sur l'impact de l'histoire naturelle sur la conception de la nature des encyclopédistes, voir James Llana, «Natural History and the Encyclopédie», Journal of the History of Biology, 33, 2000, 1-25.) De ce point de vue, Diderot semble occuper une position mitoyenne entre ces deux pôles : d'une part, cherchant à caractériser le plus objectivement possible les particularités des nations, mais, d'autre part, se laissant par moments emporter par des élans plus lyriques au sujet de certains peuples «sauvages» : voir les articles «Bédouins» et «Scythes», notamment, où ces deux attitudes sont présentes. 
sur celles des Arabes et des Juifs et sur celles des Japonais et des Chinois, pour ne citer que ces exemples, où Diderot essaie de montrer qu'il est possible que, dans chacun de ces groupes, une philosophie nationale soit un sous-produit d'une autre ou que des effets de contamination soient responsables de leur formation particulière. Il y a donc une histoire universelle de la philosophie sur le fond de laquelle Diderot parvient à penser l'altérité ethnologique. L'intérêt de cette approche est qu'elle forme un mode discursif qui non seulement met en échec les prétentions de la connaissance à accéder à l'essence de l'autre, mais qui tend aussi à nier que cette altérité puisse relever d'une essence. Elle ne peut, en effet, qu'être un produit historique. Le travail encyclopédique, alors, vise à consigner les traces de cette diversité comme autant de lieux découverts par l'être humain, au même titre que les pyramides, pense Diderot, sont "des monuments de leur prudence et du prix inestimable qu'ils attachaient à la conservation de leurs connaissances ${ }^{19}$.»

\section{Identité de l'autre}

Un recensement de ce qui, dans les histoires particulières des nations, offre une certaine récurrence montre que le fait de cette histoire universelle est lui-même soumis à certaines lois qui permettent de la comprendre.

L'article «Éthiopiens» montre en raccourci ce qu'il en est ${ }^{20}$. «On attribue, écrit Diderot, aux Éthiopiens l'invention de l'Astronomie et de l'Astrologie.» Les Éthiopiens, rencontrant les irrégularités des corps célestes, «formèrent des conjectures sur la nature de ces êtres.» Naturellement, donc, et cela justement parce que leur environnement propre le permettait, les Éthiopiens ont suivi la marche obligée de toute science et, partant, de toute philosophie, qui consiste à observer les phénomènes, puis à les rationaliser en conjectures. Le moment de déclin survient lorsqu'ils attribuent à ces corps célestes différents effets, faisant passer l'astronomie au rang d'une astrologie, dont l'article «Arabes» nous dit

19 Article «Égyptiens». M. Pinault («"Caractères et alphabets de langues mortes et vivantes" dans les planches de l'Encyclopédie», Corps écrit, 36, 123-129) écrit ainsi de ces planches qu' «elles sont aussi l'un des témoignages de la tentative des Occidentaux de vouloir conserver la mémoire des hommes de l'Antiquité comme ceux des pays lointains.» (p. 129)

20 Le choix de cet article n'est dû qu'à son caractère exemplaire. On retrouve la même stratégie argumentative dans tous les articles d' «Histoire de la philosophie». 
qu'il s'agit d'une «science vaine et ridicule». Le déclin survient en effet toujours au moment où une conjecture se change en superstition, au moment où une conjecture est mobilisée comme un savoir avéré. Dans son article sur les «Chaldéens», Diderot écrit en effet, pour expliquer leur expulsion de Rome : «La raison qu'on en donnait, c'est qu'ils abusaient de la prétendue connaissance qu'ils se vantaient d'avoir du cours des astres, pour tromper des esprits faibles et crédules, en leur persuadant que tels et tels événements de leur vie étaient écrits dans le ciel.» (Je souligne.)

Le schéma historique impliqué ici, mais qui est celui systématiquement employé par Diderot, propose une philosophie de l'histoire à deux dimensions. La première tient dans cette portion qui permet de saisir l'émergence d'une "culture» éthiopienne singulière et effective à partir de signes interprétables, de documents avérés et de témoignages. De ce point de vue, la reconstruction conjecturale de l'histoire de la philosophie des Éthiopiens dispose ces signes ou données de l'expérience de manière à rendre compte de ce qui a produit la singularité de cette philosophie et à l'inscrire, par le fait même, dans une histoire universelle.

La seconde dimension vise, pour sa part, quelque chose d'autrement plus universel, à savoir, rendre compte du phénomène de l'historicité en tant que tel et des lois qui le conditionnent, c'est-à-dire de son universalité. La description de cette seconde dimension appelle un troisième mode discursif dans le traitement de l'altérité ethnographique qui renvoie au problème de la superstition. Je l'aborde ici en guise de conclusion.

Les articles conjecturant sur l'origine d'un mythe ou d'une "philosophie» obéissent pour la plupart à un schéma à chaque fois identique. Ce fait place Diderot dans une étrange posture sur le plan de la philosophie del'histoire, puisque ce schématisme semble autant fonder, pour reprendre la terminologie de Bertrand Binoche ${ }^{21}$, une «histoire universelle» voltairienne - une histoire des faits, donc, un véritable chaos, une quasi-errance où seules les lumières théoriques font quelques progrès ${ }^{22}$ - qu'une histoire nettement plus rationalisée, où les parcours des sociétés sont présentés selon un schéma stable. Diderot, en effet, écrivant son histoire de la philosophie, conjecture sur ce qui a pu mener à la

21 Voir Les Trois sources de la philosophie de l'histoire. Paris, PUF, 1994, Première partie, chapitre 2 : «L'Histoire universelle», 33-52.

22 Voir Éliane Martin-Haag, Voltaire. Du cartésianisme aux Lumières, Paris, Vrin, 2002, Deuxième partie, chapitre $1:$ :Le problème d'une philosophie de l'histoire», 105-116. 
formation des idées et des mœurs des différents peuples. Mais, en tentant de respecter les particularités propres aux différentes nations, Diderot, au fond, conte toujours à peu près la même histoire, histoire qui correspond à ce cycle de vie auquel tout corps est soumis ${ }^{23}$ : naissance, développement, stagnation ou mort, histoire, donc, qui correspond à la théorie cyclique de l'évolution des sociétés développée par nombre de penseurs du XVIII ${ }^{\mathrm{e}}$ siècle, dont Helvétius n'est pas le moindre. ${ }^{24}$

Il est primordial de rappeler que ce schéma ne peut jamais être compris que comme une conjecture. Il vise à montrer qu'il y a une régularité à l'œuvre dans les histoires des nations - une régularité faisant office de loi. En la circonstance, on pourrait dire que le sens de ce qu'est une loi historique, pour Diderot, suit la ligne dure du newtonianisme de son temps ${ }^{25}$, $c^{\prime}$ est-à-dire qu'il ne saurait être question de penser cette loi en termes de réalisme ontologique. Le schéma tracé par Diderot a pour seule fonction de rendre compte des phénomènes. Mais cette interprétation des données offertes par l'«histoire universelle» a déjà un caractère plus philosophique, puisqu'elle offre une histoire réfléchie où se découvre un principe anthropologique. Ce principe, c'est celui qu'on voit à l'œuvre dans l'article "Éthiopiens" cité plus haut, à savoir la tendance de toute conjecture explicative à tourner en superstition, par le jeu de la vanité de celui qui croit vraie sa conjecture, à fonder des mœurs sur cette superstition qui en font oublier le caractère partiel et approximatif. Tendance qui n'est autre qu'une prétention à nier la valeur du discours de l'autre. Tendance, de même, qui n'est autre que celle à s'arroger un pouvoir despotique sur autrui. Là est le lieu précis où se croisent superstition, dogmatisme et despotisme, religion, mœurs et politique.

23 La plupart des commentateurs sont d'accord sur ceci que coexistent dans la pensée de Diderot l'idée de progrès et une théorie cyclique de l'histoire. Voir J. Proust, op. cit., p. 447-448 et G. Benrekassa, «La pratique philosophique de Diderot», Stanford French Review, automne 1984, 189-213.

24 Sur cette conception de l'histoire, voir Michèle Duchet, op. cit., chapitres «L'anthropologie d'Helvétius» et «L'anthropologie de Diderot», p. 377-476.

25 On sait que Diderot connaît bien Newton, pour être passé proche, au temps où il travaillait beaucoup sur les mathématiques, à peine quelques années avant de commencer à travailler sur l'Encyclopédie, d'écrire un ouvrage d'introduction à la théorie newtonienne. Voir Lester Gilbert Krakeur et Raymond Leslie Krueger, «The Mathematical Writings of Diderot», Isis, juin 1941, XXXIII, p. 219-232 et Aram Vartanian, "Diderot et Newton" dans Claude Blanckaert, Jean-Louis Fischer et Roselyne Rey, Nature, histoire, société. Essais en hommage à Jacques Roger, Klincksieck, 1995, 61-78. 
Ainsi, la description de la formation des superstitions des autres nations prend, par le détour d'une inscription de cette formation dans un schéma général explicatif de toute l'histoire humaine, une valeur de parallèle réflexif faisant retour sur le lecteur européen de l'Encyclopédie qui se voit ainsi forcé de penser sa propre propension au dogmatisme ou à la superstition ${ }^{26}$. Ce qui explique, dès lors, la nécessité pour Diderot de toujours recourir à une neutralisation du savoir ethnographique qu'il mobilise, pour empêcher qu'il ne tourne en dogme ${ }^{27}$.

Trois modes discursifs, donc : un mode sceptique par lequel Diderot force l'histoire à entrer au rang de science purement conjecturale ; un mode factuel magnifiant la diversité possible au sein de l'espèce ; et un mode plus réflexif où le philosophe tente de saisir les lois de l'histoire. Ces trois modes sont solidaires de cette manière diderotienne d'envisager la nature de son travail - qu'il soit encyclopédique, littéraire ou philosophique - à savoir, avant tout, de "changer la façon commune de penser», de "faire penser» son lecteur, de maintenir la pensée en mouvement, ultime effort contre cette tendance à l'inertie de la pensée.

\section{MITIA RIOUX-BEAULNE}

Université de Montréal

26 Ainsi, concernant le rapport de Diderot à la religion juive, D. Bourel («Les rasés et les barbus : Diderot et le judaïsme», Revue philosophique de la France et de l'étranger, 1984, no. 4, p. 275-285) note que celui-ci attaque le judaïsme en vue de discréditer les apologistes de la religion juive au moins autant en vue d'atteindre la religion chrétienne. T. Scanlan repère exactement le même procédé dans les articles portant sur la religion musulmane ( Islam and its Founder as Presented in Diderot's Encyclopédie», Studi francesi, 112, 1994, 11-21.) Un dernier exemple de cette introduction de parallèles est donné par H. Nakagawa ( «L'Encyclopédie de Diderot et le Japon» dans Colloque international Diderot, Paris, Aux Amateurs du Livre, 1985, 411-421) qui montre que dans l'article "Japon", Diderot raconte l'histoire d'une secte confucianiste éclairée qui rappelle très exactement les déboires qu'il connaît en tant que directeur de l'Encyclopédie.

27 J. Starobinski fait remarquer que les stratégies discursives à l'œuvre dans $l^{\prime}$ Encyclopédie ont justement la vertu de transformer «en une sorte de dialogue avec le lecteur ce qui d'abord se présentait comme la séquence monologuée d'une vaste série de monographies savantes.» («Remarques sur l'Encyclopédie», Revue de métaphysique et de morale, 1970, p. 284-291 [p. 287 pour la citation].) 